\title{
18 \\ Large Scale Multi-User Virtual Worlds
}

\author{
Gurminder Singh, Tapas K. Das \\ Institute of Systems Science, National University of Singapore \\ Kent Ridge, Singapore 119597, REPUBLIC OF SINGAPORE \\ Phone: +65 772-3651, Fax: +65 774-4998, Email: gsingh@iss.nus.sg
}

\begin{abstract}
We describe how WorldNet, a software toolkit for networked virtual worlds, solves the problem of scaling up to support large numbers of simultaneous virtual world users. The two key ideas behind our approach are to partition virtual world users into groups and to use Group Dead-Reckoning technique. These two techniques combined together reduce the total network bandwidth required as well as the computation power required at the virtual world client to retrieve and process network messages.
\end{abstract}

\section{Keywords}

Key Words: Networked Virtual Reality, Distributed Interactive Environments, Multiuser Virtual Worlds, Client-Server Computing, Distributed Interactive Simulation.

\section{INTRODUCTION}

Over the last 2-3 years, several groups around the world have initiated research and development projects in networked virtual worlds. These efforts aim to develop software infrastructure to support multiple users to share information with one another through virtual worlds connected over networks. Most of these systems can support a few users efficiently, but when the number of users becomes larger than a handful, the overall performance of the network and the associated virtual worlds quickly becomes unbearably sluggish.

Our effort is aimed at supporting large numbers (several hundred) of simultaneous virtual world users competing, collaborating or playing with one another. Our users' environment consists of high-end PCs (at least $90 \mathrm{MHz}$ Pentium with 16MB RAM). The servers that support these users run on SUN SPARCstation 10 and 20 workstations. These servers and clients are connected over internet.

In this paper we describe problems associated with a scalable architecture and explain how a distributed server-based solution can solve these problems. 


\section{A SAMPLE APPLICATION - GROUP TREASURE HUNT}

We have developed a group treasure hunt game that we have tested with 100 simultaneous users with great success. This game is played on INTEL Pentium PCs connected on internet. Soon we intend to expand our test to include about 200 users. Our game is quite different from the common "twitch" or arcade-type games in the sense that it is not a short-duration, intense, hand-eye coordination game. An instance of our game, once started, should run for a few (4-6) weeks; players can join and leave the game at their convenience.

This game involves searching for a treasure which is hidden in a complex maze of rooms and corridors. The maze is large enough that it is easy to get lost in it. The rooms of the maze have hurdles that the players have to overcome. One of the most common hurdles is to find the key that opens the door so that other parts of the maze connected to the room can be explored. Keys are hidden in walls, floors and ceilings of rooms and in furniture found in rooms. Rooms have "televisions" that, on demand, provide helpful hints to players to follow.

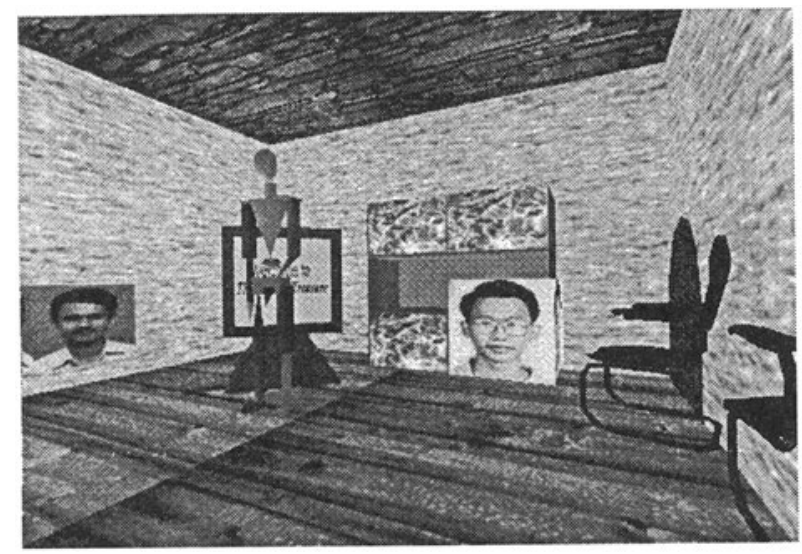

Figure 1 A sample screen from the Group Treasure Hunt game. Players can see one another in the game environment.

The players are partitioned into groups of 20 each. Within a group, the players collaborate with each other, whereas across groups players compete. Collaboration within a group happens by sharing with each other things that a player finds in the maze, by exploring different paths in the maze, by hiding things so that opponents would have difficulty in finding them, by attacking opponents collectively, by saving group members from attacks by other groups, and by sharing information with the other members of the group.

During play, members of a group can see one another in the environment (see figure1) as well as in the outline of the maze. Actions which are global can also be seen by the other players. Players from one group begin to see rival groups when their groups have attained a certain level of expertise in the game. Here again, only those rival groups are visible which have attained the same or higher level of expertise. This way new groups are protected from experienced groups until they are ready to "deal with the real world".

In the following paragraphs we briefly describe how WorldNet ${ }^{1}$ architecture supports this 


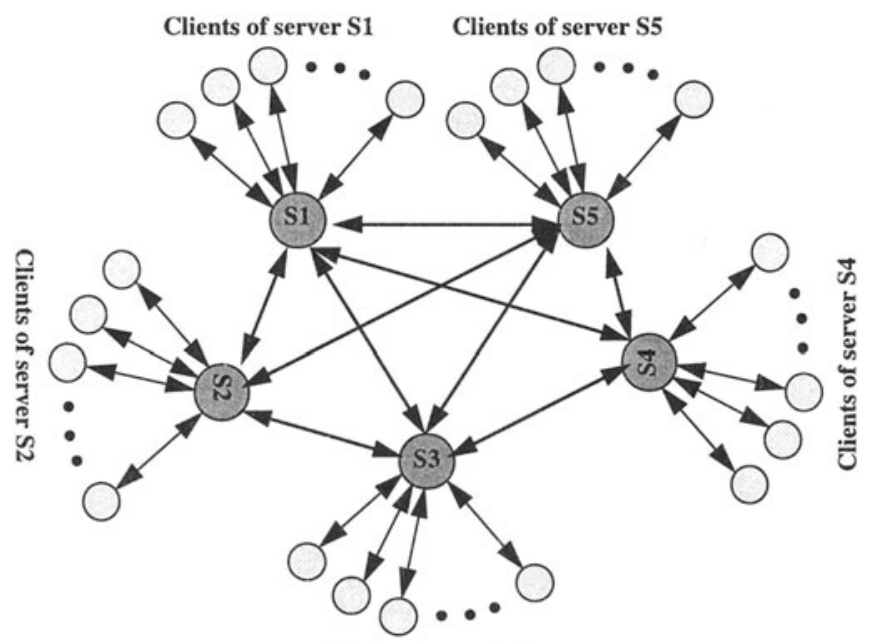

Clients of server S3

Figure 2 Architecture of WorldNet. A Worldnet server can handle a group of Group Treasure Hunt game players, allowing tight communication within a group. Servers cooperate with one another to support communication across groups.

game. Detailed description of the WorldNet architecture can be found in (Singh et al., 1994) and (Singh et al., 1995). WorldNet uses distributed, co-operating servers and message filtering (see Figure 2) to

- $\quad$ reduce the number of messages that have to be distributed, thus reducing the network bandwidth required, and

- reduce the messages that the clients have to process, thus reducing the computational power required.

For this game each server (possibly running on a separate workstation) handles one group of players. This makes it very efficient for the server to handle communication within the group. For communication across groups, respective group servers communicate with each other, which in turn communicate with their clients.

When players join the game, they all connect to a single server. On connection, a player is asked to select a group he or she would like to join. Depending on the selection, the player is migrated (transparently) to the WorldNet server handling the group.

In addition to multiple servers, a "group dead-reckoning" method is used to eliminate sending messages each time a player moves. Players send their positions to their server at regular intervals. Instead of broadcasting the position of each individual player to the whole group, the group server accumulates position updates from each player and broadcasts one big packet at regular intervals to the whole group (see Figure 3). This packet contains each client's current position and velocity vector. On receiving this packet, a player updates the position of its group members.

1. WorldNet is the networking part of BrickNet. Bricks constitutes the graphical part of BrickNet. 
From then on, it uses the velocity vector to predict the position of individual players, until the next packet is received. Group dead-reckoning used in this game has two main effects:

- $\quad$ it reduces the number of updates sent by a player

- $\quad$ it reduces further the number of updates sent by the server to its group.

Further details of how server migration is implemented and how players messages are combined is provided in section 4.2.

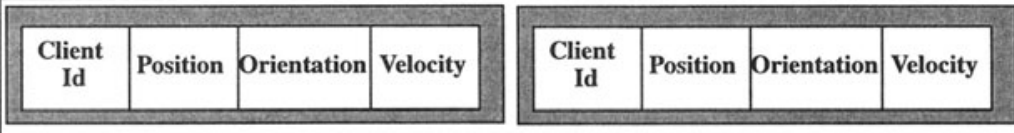

Figure 3 Format of the Group Dead-reckoning update packet.

\section{RELATED WORK}

Most existing networked virtual world systems can support only a very small number (10 users is considered high) of simultaneous users. SIMNET (Calvin et al., 1993), NPSNET (Macedonia et al, 1995) and RING (Funkhouser, 1995) are clearly in a different class when it comes to number users - these systems have aimed to support a much larger number of simultaneous users than most other systems. SIMNET and its descendent, DIS (IEEE), can support about 300 users, whereas NPSNET is gearing up to support more than 1000 users (Macedonia et al., 1995a). Instead of covering all important networked virtual world systems here, we will cover only SIMNET, DIS, NPSNET and RING. Information on other interesting networked virtual world systems can be found in VR-DECK (Codella et al., 1993), DIVE (Carlsson \& Hagsand, 1993), EM (Wang, Green \& Shaw, 1995) and MASSIVE (Greenhalgh \& Benford, 1995).

SIMNET uses an object- and event-based approach to distributed, interactive virtual worlds for battlefield simulation and training. In SIMNET, virtual worlds consist of objects that interact with each other by broadcasting a series of events. An object initiating an event does not calculate which other objects might be affected by it. It is the receiving object's responsibility to determine whether the event is of its interest or not. To reduce communication processing and bandwidth requirements, objects transmit update messages at a fixed regular interval. Until an update is received, the new position of a remote (or a network-based) object is extrapolated from the states last reported by those objects.

Distributed Interactive Simulation (DIS) is a descendent of SIMNET and is an emerging IEEE standard. Both SIMNET and DIS use broadcast for communication among virtual worlds. All objects, static or mobile, must send update messages at regular intervals to inform others of their current state - whether or not their state has changes, and whether or not the receiving client would be affected.

NPSNET uses a combination of DIS and IP Multicast for communication among virtual worlds. By using spatial, functional and temporal relationships among virtual world objects, NPSNET forms multi-cast groups (Macedonia et al., 1995a). Objects can belong to several multicast groups at a time. This partitioning enables NPSNET to reduce the bandwidth and computational requirements of virtual worlds. By using multicast groups, NPSNET aims to reduce the impact of some of the most serious problems associated with using the DIS protocol to support 
large numbers of users.

RING is a client-server-based system that supports interaction among large numbers of users in virtual environments with dense occlusions. It uses server-based visibility algorithms to compute potential visual interaction among entities in order to reduce the number of messages required to maintain consistent state among virtual world clients. Based on this computation, state changes are propagated only to hosts containing entities that can possibly perceive the change.

\subsection{Relation to WorldNet}

SIMNET and DIS use broadcast for communication among virtual worlds whereas WorldNet uses a distributed server approach. While broadcast as used in SIMNET and DIS can possibly lead to a more continuous updates, specially in the case of a small number of clients, it can quickly bog down virtual worlds with too many network messages. WorldNet, on the other hand, can scale up to handle a much larger number of users by allocating a smaller number of users to each server and having many servers. WorldNet extends the idea of dead-reckoning to take advantage of its distributed server architecture. Group dead-reckoning is explained in section 4.1.

NPSNET has evolved from a broadcast-based approach to a multicast-based approach. NPSNET's approach of using spatial, functional and temporal relationships among virtual worlds to create multi-cast groups maps very nicely to WorldNet's distributed servers. Each distributed server can be seen as a multicast group, the main difference being that servers, since they are workstations can do filtering and grouping of messages that is not possible with NPSNET's multicast groups. In principle, WorldNet should be able scale up to handle larger number of simultaneous users than NPSNET but this needs to be verified with a real-life experiment.

RING, like WorldNet, is a client-server-based system; SIMNET and DIS are broadcast systems whereas NPSNET is a multicast-based system. RING uses visibility calculation to reduce the number of messages that are sent in the system. WorldNet uses semantic information about objects to minimize network message. When all virtual worlds are not identical in their content, WorldNet forwards messages based on the objects that the clients have (see (Singh et al., 1994) for further discussion on how this is achieved). For virtual worlds with identical content, WorldNet uses distributed servers combined with group dead-reckoning to reduce the network traffic (explained later in this paper). RING's approach could be used to further reduce network messages in WorldNet.

\section{REDUCING NETWORK AND COMPUTATIONAL REQUIREMENTS}

The two important issues that one has to deal with in supporting a large number of simultaneous user in networked virtual worlds are:

- $\quad$ reducing the overall network bandwidth required for communication among virtual worlds, and

- $\quad$ reducing the CPU cycles spent by a virtual world client on handling (both sending and receiving) network messages.

From an overall perspective, it may appear that these two issues actually translate to one issue only: if we were to reduce the time spent in sending and receiving messages, we would in effect reduce the total bandwidth required. While this is true, it makes better architectural sense if we treat these two issues separately. It is possible to develop networked virtual worlds where the network can support many more users but the individual workstations of the users get swamped by 
the network traffic to such an extent that they have little time to process graphics. On the other hand, it is possible to have workstations which have plenty of spare cycles but the network cannot efficiently support them. To deal with both these scenarios, we need to work on the above two issues separately as well as together.

In the following two sections, we describe how WorldNet addresses these requirements.

\subsection{Group Dead-Reckoning}

In this section, we briefly explain how dead-reckoning works and then go into the details of group dead-reckoning that we have developed in WorldNet. For greater details on dead-reckoning see (Blau et al., 1992) and (Macedonia et al., 1994). Dead-reckoning is a per-object-based technique for periodically synchronizing object positions in multiple virtual worlds. In this technique, each object is controlled in its own virtual world by a software object called Player. In every other virtual world in the network, the Player is dynamically modelled as an object called Ghost. The Player object periodically sends its position and velocity on the network. When this update is received by the Ghost, it first corrects its position to the new position of the player and resumes predicting the position of the Player from then on until the next update is received. The primary purpose of using dead-reckoning is to reduce network traffic by minimizing updates at the cost of computation by the virtual world workstation.

In WorldNet, we have extended the concept of dead-reckoning from a per-object-based technique to a group technique. In this technique, the objects send their updates just like in simple dead-reckoning, but with two essential differences:

a) objects send updates to their respective group servers, and

b) all object updates are received together (in a single packet) by a virtual world client.

All objects, except those whose state has not changed, periodically send updates to their server (see Figure 2). But instead of forwarding updates to the group members as and when updates are received, the server gathers all updates in a single packet and sends out this update packet periodically (see Figure 3). The receiving clients can then update all objects at the same time using a suitable dead-reckoning algorithm. To make this technique work best, a good compromise has to reached between the frequency of clients sending updates to the server and the server sending updates to its clients. Currently, the clients send updates to the server twice as frequently as the server sending the update to clients. This ensures that the update received by the clients is the most recent update. The two advantages group dead-reckoning has over simple deadreckoning are that"

- There are fewer messages sent from the server to the clients, thus reducing the network bandwidth required. Also, internet, our main delivery platform, is lot more efficient with sending one large packet than many small packets.

- The clients have to receive and process fewer messages. A client basically receives one big packet that contains all updates, compared to receiving and processing each update as a separate packet.

\subsection{Distributed Servers}

Distributed servers, as used in WorldNet, reduce the overall network bandwidth required by basically reducing the number of clients to whom the updates are sent. This is achieved by first identi- 
fying a group of clients whose updates affect one another and then migrating these clients to a common group server. The basis for forming groups can be spatial, temporal or functional. For the Group Treasure Hunt game described in section 2, grouping is based on functional requirements (The members of a group cooperate and share information with one another and they gang together to compete with other groups). Individual clients send their updates to their group server, which combines several updates in a single packet and sends this packet to all members of the group.

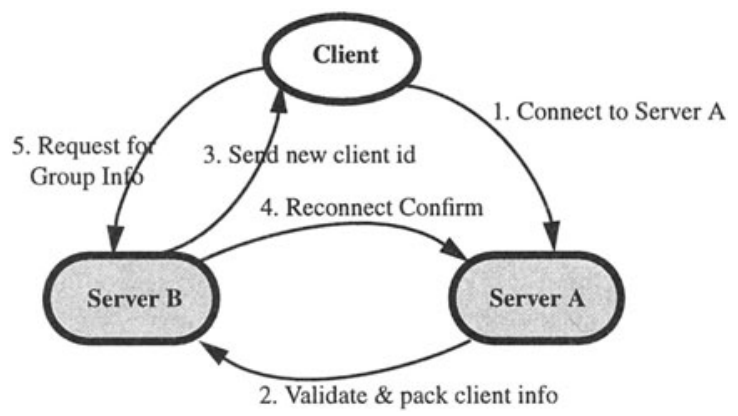

Figure 5 Server migration in WorldNet.

An important part in using the distributed server approach is the ability to migrate clients from one server to another transparently when groups are formed. The server migration process in WorldNet is shown in Figure 5. When a client is to be moved from Server A to Server B, all its object information needs to be moved as well.

When moving a client from Server A to Server B, Server A first packs the relevant client information and makes a contact with Server B. Upon receiving a reconnect request, Server B retrieves the client information and attempts to establish contact with the client. Once this contact has been established, Server B sends a reconnection OK message back to Server A. At this time, Server A releases the client. The client then requests for relevant information from Server B and the migration is completed.

\section{CONCLUSIONS}

We have presented a highly-scalable software architecture for networked virtual worlds. There are two main ideas behind this architecture. The first idea is to divide virtual world users into groups and allocate one server per group. The second idea is to use group dead-reckoning. These two ideas combined together can reduce both the network bandwidth required as well as the computation power required at the client workstation to process network messages. We have developed a Group Treasure Hunt game based on this architecture and are in process of testing it. Our initial experiments have been very encouraging, giving us hope that our system would be able to cope up with a few hundred users. The users' environment for this test would be Pentium PCs, 14,400 bps modems and internet.

\section{REFERENCES}

Calvin, J. et al. (1993). The SIMNET Virtual World Architecture. Proc. IEEE VRAIS'93, Sept. 18- 
22, 1993, Seattle, Washington, USA, pp: 450-455

Carlsson, C. \& Hagsand, O. (1993). DIVE - a Multi-User Virtual Reality System. Proc. IEEE VRAIS'93, Sept. 18- 22, 1993, Seattle, Washington, USA, pp: 394-400

Codella, C., Jalili, R., Koved, L., \& Lewis, B.J. (1993). A Toolkit for Developing Multi-User, Distributed Virtual Environments. VRAIS'93, Sept. 18- 22, 1993, pp: 401-407

Funkhouser, T. (1995) RING: A Client-Server System for Multi-User Virtual Environments. Proc. 1995 Symposium on Interactive 3D Graphics, Monterey, CA, April 19-21, 1995, pp: 85-92

Greenhalgh, Chris \& Benford, Steve (1995) MASSIVE: A Collaborative Virtual Environment for Tele-Conferencing. ACM Transactions on Computer-Human Interaction, Sept. 1995.

IEEE P1278 - Standard for Information Technology - Distributed Simulation Application Process and Data Entity Interchange Formats.

Macedonia, M.R. et al. (1995) NPSNET: A Network Software Architecture for Large Scale Virtual Environments. Presence, MIT Press, Boston, 3(4)

Macedonia, M.R. et al. (1995a) Exploiting Reality with Multicast Groups: A Network Architecture for Large-scale Virtual Environments. IEEE VRAIS'95, North Carolina, USA, March 11-15, 1995, pp: 2-10

Singh, Gurminder et al. (1995) BrickNet: Sharing Object Behaviours on the Net. IEEE VRAIS'95, North Carolina, USA, March 11-15, 1995, pp: 19-15

Singh, Gurminder et al. (1994) BrickNet: A Software Toolkit for Network-Based Virtual Worlds. Presence: Teleoperators and Virtual Environments, MIT Press, Boston, 3(1), pp: 19-34

Wang, Q., Green, M. \& Shaw, C. (1995) EM - An Environment Manager for Building Networked Virtual Environments. IEEE VRAIS'95, March 11-15, 1995, pp: 11-18

\section{BIOGRAPHY}

Dr. Gurminder Singh is Program Director of Creative Services at the Institute of Systems Science, National University of Singapore and Director of Imagine Interactive Inc. For many years he has been involved in R\&D in Virtual Reality, User Interface software and Visual Languages. Much of what he has done has been either converted into commercial products or systems that people use to do their everyday work. Dr. Singh is on the editorial boards of ACM Interactions, ACM-Springer Multimedia Systems and the Advances in Human-Computer Interaction book series published by Ablex. He organized the first Virtual Reality Software and Technology (VRST 94) conference, which has since become a regular international conference. He has recently edited or co-edited the following special issues on Virtual Reality: Communications of the ACM (May 1996), ACM Transactions on Computer-Human Interaction (September 1995), and ACMSpringer Multimedia Systems(1996, forthcoming).

Tapas K Das is a Software Engineer at the Institute of Systems Science where he is involved in the Interactive Discovery Learning project. Previously he has worked at Fujitsu Singapore for 2.5 years on ISDN protocols for Digital Switching Systems. Tapas has B.Tech. in Computer Science and Engineering from IIT, Kharagpur, India. He is working on his M.Sc. in the area of networked VR systems. 